\title{
Alendronate sodium in the management of osteoporosis
}

\author{
P J J Prinsloo' \\ D J Hosking ${ }^{2}$ \\ 'Dept of Clinical Chemistry, City \\ Hospital, Nottingham, UK; ${ }^{2}$ Dept \\ Endocrinology and Diabetes, City \\ Hospital, Nottingham, UK
}

\begin{abstract}
Alendronate is one of the best and most extensively studied bisphosphonates in the treatment of osteoporosis. This review considers in detail the major pivotal study, the fracture intervention trial (FIT), upon which the use of alendronate is based and which was a landmark study in terms of design, size and clinical impact. The role of alendronate has subsequently been underscored by a range of studies extending the clinical indications for its use and consolidating the effect on reducing both vertebral and non-vertebral fracture risk. Although the emphasis of these studies has predominantly been on the management of postmenopausal osteoporosis, data is also available in primary prevention, men, and glucocorticoids-induced osteoporosis. Direct comparison between the different drugs used to treat osteoporosis with fracture end points are needed for patients and doctors to make informed choices, but the size of such studies are prohibitive. Clinical trials using surrogate markers such as bone mineral density and biochemical markers of bone turnover have been performed which provide some helpful information but the limitations of this approach need to be recognized.
\end{abstract}

Keywords: alendronate, osteoporosis, hormone replacement therapy, male osteoporosis, parathyroid hormone, corticosteroid-induced osteoporosis

\section{Introduction}

Alendronate is one of the best and most extensively studied bisphosphonates in the treatment of osteoporosis. The fracture intervention trial (FIT) was a landmark study in terms of design, size, and clinical impact. The role of alendronate has subsequently been underscored by a range of studies extending the clinical indications for its use and consolidating the effect on reducing both vertebral and non-vertebral fracture risk.

\section{Pivotal studies}

A quartet of pivotal studies formed the basis for the subsequent clinical trials of alendronate and for post hoc analyses (Liberman et al 1995; Black et al 1996, 2000; Cummings et al 1998). The first of these examined the dose-response relationship in terms of gain in bone mineral density (BMD) and fracture risk reduction but a more extensive study of the effect on fractures was the central feature of the Fracture Intervention Trial (FIT). They will be considered in detail since they are of such importance to the subsequent use of alendronate in the treatment of osteoporosis.

\section{Alendronate dose-response (Liberman et al 1995)}

One of the great strengths of the alendronate clinical research programme was the early demonstration of a clear dose-response relationship and the identification of $10 \mathrm{mg}$ daily as the optimal dose in terms of the gain in BMD. In this trial, 994 women 
with postmenopausal osteoporosis (lumbar spine BMD T Score $<-2.5$ ), of whom about $20 \%$ had a prevalent vertebral fracture, were randomized to placebo, $5 \mathrm{mg}$ or $10 \mathrm{mg}$ daily for three years, or $20 \mathrm{mg}$ daily for two years, and then $5 \mathrm{mg}$ daily for the third year. It was a well conducted investigation with $91 \%$ of the women completing a year of the study and paired spine films being available on 97\%. After 3 years, the $10 \mathrm{mg}$ dose had increased lumbar spine BMD by $8.8 \%$ relative to placebo with increments of $5.9 \%$ and $7.8 \%$ at the femoral neck and trochanteric area of the hip with a gain of $2.5 \%$ at the total body and $2.2 \%$ at the mid forearm $(\mathrm{p}<0.001$ for all comparisons). The $10 \mathrm{mg}$ dose was significantly more effective than the $5 \mathrm{mg}$ dose at all skeletal sites, but was as effective as the $20 \mathrm{mg}$ dose. Most of the gain in BMD occurred in the first 6 months of treatment but was independent of the baseline value.

The relative risk (RR) of a new morphometric fracture among women treated with alendronate (all doses combined) compared with placebo was 0.52 (95\% confidence index [CI] 0.28-0.95). Interestingly the protective effect was independent of alendronate dose, presence of a previous vertebral fracture or age $>65$ years. Multiple vertebral fractures were also reduced, although absolute numbers were small. The Spinal Deformity Index (SDI) an aggregate of vertebral deformity showed a similar pattern of response with protection afforded by alendronate but of marginal statistical significance $(p=0.054)$. Alendronate also reduced the risk of height loss by $35 \%$ which was more marked in women $\geq 65$ years and in those with a prevalent fracture. There was no effect on height loss in women without a new vertebral fracture, but in those with a new vertebral fracture, alendronate reduced height loss (placebo: $23.3 \mathrm{~mm}$, alendronate: $5.9 \mathrm{~mm}$ ). This latter effect represents the action of alendronate to decrease the number and severity of wedge and crush fractures. There was a trend towards a reduction in non-vertebral fractures but this did not reach statistical significance (RR 0.79, 95\% CI 0.52-1.22) and since this was a post-hoc analysis it requires caution in interpretation. Alendronate was well tolerated with no excess of drug-related withdrawals, severe adverse events, or discontinuation due to upper gastrointestinal effects.

This was an important study of patients who were not selected because they were at high risk of fractures and who were probably more representative of the usual clinic population.

\section{FIT: vertebral fracture arm (Black et al 1996)}

The FIT comprised two studies with the pre-specified aim that the combination would be analyzed as well as the individual arms. The first study to be reported included 2027 women aged 55-81 years with a prior vertebral fracture and a femoral neck BMD $<0.68 \mathrm{~g} / \mathrm{cm}^{2}$, considered to be equivalent to a value of 2.1 standard deviations (SD) below the young normal peak BMD (T score). Subsequent to the start of FIT the results from the Third National Health and Nutrition Examination Survey (Looker et al 1995) had shown that the BMD inclusion criteria corresponded to a $\mathrm{T}$ score of -1.6 , much higher than the intended value of -2.1 but still consistent with a diagnosis of osteoporosis in women with a prior vertebral fracture. Study subjects were randomized either to alendronate (1022) or placebo (1005).The alendronate dose was $5 \mathrm{mg}$ daily for the first two years and then $10 \mathrm{mg} /$ day after the Phase III study (Liberman et al 1995) showed the superiority of the larger dose in terms of gain in BMD and reduction in bone turnover. A history of (any) fracture since the age of 45 years was given by $58 \%$ of the placebo group and $57 \%$ of those given alendronate. Calcium intake was assessed by food frequency questionnaire and those with an intake $<1 \mathrm{~g} /$ day (82\%) were given supplements of $500 \mathrm{mg}$ calcium and $250 I U$ vitamin D. The primary end point of the trial was a new morphometric vertebral fracture defined as a reduction of $20 \%$ (and at least $4 \mathrm{~mm}$ ) in either anterior, mid, or posterior vertebral height between baseline and last follow up visit. Secondary end points included any clinical fractures and loss of height. Clinical fractures were defined as one diagnosed by a physician while self-reported fractures were confirmed by a written radiographic report or other tests. The trial entrants were predominantly (97\%) Caucasian and spine radiographs were obtained at the close out visit in 1949 out of 2027 patients. At the close out visit, 90\% of the women were still taking the trial medication and $96 \%$ of them had taken $>75 \%$ of their pills since the previous visit. This was a very well conducted trial, providing good quality fracture data.

The results are summarized in Table 1 but included a $47 \%$ reduction in morphometric fractures (primary end point), a 55\% reduction in clinical vertebral fractures and a $28 \%$ reduction in clinical spine and non-spine fractures (main secondary end point). These effects were independent of baseline BMD. There was not a significant reduction in all non-spine fractures although wrist and hip fractures were 
Table I Fracture risk in postmenopausal women with a prevalent vertebral fracture*

\begin{tabular}{llll}
\hline Fracture type & Placebo (\%) & Alendronate (\%) & Fracture risk (95\% Cl) \\
\hline Morphometric $\geq I$ & $145(15)$ & $78(8)$ & $0.53(0.4 I-0.68)$ \\
Morphometric $\geq-2$ & $47(4.9)$ & $5(0.5)$ & $0.10(0.05-0.22)$ \\
Clinical vertebral & $50(5.0)$ & $23(2.3)$ & $0.45(0.27-0.72)$ \\
Any clinical & $183(18.2)$ & $139(13.6)$ & $0.72(0.58-0.90)$ \\
Any non-vertebral & $148(14.7)$ & $122(11.9)$ & $0.80(0.63-1.01)$ \\
Hip & $22(2.2)$ & $11(1.1)$ & $0.49(0.23-0.99)$ \\
Wrist & $4 I(4.1)$ & $22(2.2)$ & $0.52(0.31-0.87)$ \\
Height loss $(\mathrm{mm})$ & 9.3 & 6.1 & $\mathrm{P}<0.001$ \\
\hline
\end{tabular}

Note: * Black et al 1996.

Abbreviations: $\mathrm{Cl}$, confidence interval.

significantly reduced as was height loss. The gain in BMD, relative to placebo, was similar to that seen in the Phase III study and was $6.2 \%$ at the lumbar spine, $4.1 \%$ at the femoral neck, $4.7 \%$ at the total hip, $6.1 \%$ at trochanter, $1.8 \%$ at total body, and $1.6 \%$ at proximal forearm.

Adverse events including those arising from the upper gastrointestinal tract (placebo 2.2\%, alendronate 1.6\%) were similar between alendronate and placebo and this was also true in the third year when the alendronate dose was increased from $5 \mathrm{mg}$ to $10 \mathrm{mg}$ daily. There was no excess of adverse events in either group in women taking concomitant non-steroidal anti-inflammatory drugs.

\section{FIT: clinical fracture arm (Cummings et al 1998)}

Two years later the effects of alendronate on fracture risk in postmenopausal women with a low BMD, but no vertebral fracture was reported in 4432 women aged 54-81 years with a femoral neck BMD $<0.68 \mathrm{~g} / \mathrm{cm}^{2}$. As discussed above, the Third National Health and Nutrition Examination Survey (NHANES III) (Looker et al 1995) had shown that the BMD inclusion criteria corresponded to a $\mathrm{T}$ score of -1.6 rather than the intended value of -2.1 . A total of 2218 women were randomized to placebo and 2214 to alendronate and followed for an average of 4.2 years. Although the entry requirement was the absence of a prior vertebral fracture, $35 \%$ of those receiving placebo and $36 \%$ given alendronate had a history of a non-spine fracture since the age of 45 years which was about $20 \%$ less than that seen in the vertebral fracture study. Otherwise the two trial protocols were identical, including the increase in alendronate dose at two years, the provision of calcium and vitamin D supplements, compliance with trial medication and the completeness of data acquisition.

The gain in BMD at 4 years with alendronate relative to placebo (bone gain with treatment + bone loss with placebo) was similar to previous studies: lumbar spine 6.6\%, femoral neck $4.6 \%$, trochanter $6.8 \%$, total hip 5.0\%, total body $2.0 \%$, and ultra-distal radius 3.1\%. The increase in BMD was independent of baseline BMD. Although alendronate reduced the risk of most types of fracture by about $12 \%$ $21 \%$ none of these changes were significant (Table 2). The exceptions were morphometric vertebral fractures and "other clinical fractures" (which excluded clinical fractures of spine, hip and wrist) but showed a $21 \%$ reduction relative hazard (RH) 0.79 , 95\% CI 0.65-0.96). The risk of wrist fractures appeared to increase. The effect of treatment was dependent on baseline BMD so that it was only patients with a femoral neck BMD below -2.5 based on NHANES III (lowest tertile) who showed a significant protective effect.

Table 2 Fracture risk in postmenopausal women without a prevalent vertebral fracture*

\begin{tabular}{llll}
\hline Fracture type & Placebo (\%) & Alendronate (\%) & Fracture risk (95\% Cl) \\
\hline Morphometric $\geq 1$ & $78(3.8)$ & $43(2.1)$ & $0.56(0.39-0.80)$ \\
Morphometric $\geq 2$ & $10(0.5)$ & $4(0.2)$ & $0.40(0.13-1.24)$ \\
Clinical vertebral** & & & \\
Any clinical & $312(14.1)$ & $272(12.3)$ & $0.86(0.73-1.01)$ \\
Any non-vertebral & $294(13.3)$ & $261(11.8)$ & $0.88(0.74-1.04)$ \\
Hip & $24(1.1)$ & $19(0.9)$ & $0.79(0.43-1.44)$ \\
Wrist & $70(3.2)$ & $83(3.7)$ & $1.19(0.87-1.64)$ \\
Height loss $(\mathrm{mm})$ & 8.5 & 7.0 & $\mathrm{P}<0.001$
\end{tabular}

Note: *Cummings et al 1998, **Clinical vertebral fractures included in "any clinical" fractures.

Abbreviations: $\mathrm{Cl}$, confidence interval. 
In this subgroup, clinical fractures were reduced by $36 \%$ (RH 0.64; 95\% CI 0.50-0.82), hip fractures by 56\% (RH 0.44; 95\% CI 0.18-0.97) and morphometric spine fractures by $50 \%$ (RH $0.50 ; 95 \%$ CI $0.31-0.82$ ), but there was no reduction in wrist fractures. Adverse events were similar between placebo and alendronate as had been found previously.

This study shows an interesting contrast between the gain in BMD which was independent of starting value and the reduction in fracture risk which was strongly influenced by a BMD below -2.5 . This is consistent with an effect of alendronate reducing the size of the remodelling space in patients with thinned trabeculae as shown by the low BMD (Heaney et al 1997). The importance of reducing the first fracture with anti-resorptive therapy is the hope that it will reduce the risk of future fractures which is strongly influenced by the first event (Klozbuecher et al 2000).

\section{FIT: osteoporotic cohort (Black et al 2000)}

The observation from the previous study that fracture risk reduction only occurred in about a third of the cohort (1631/ 4438) with osteoporosis, as shown by a femoral neck T score below -2.5 , reinforced the need which had been prespecified in the FIT data analysis plan to compare and pool the osteoporotic women in the two arm of FIT. Before pooling studies, it is necessary to show the homogeneity of the odds ratio with treatment and this was confirmed by the Breslow-Day test. The baseline characteristics of the two groups were similar except that the women without vertebral fractures were about 2 years younger, less likely to have had a non-spine fracture since age 45 years, but had a lower hip (but not spine) BMD. Vertebral and non-vertebral fracture rates were higher in the women with a prior vertebral fracture as would be expected (Klozbuecher et al 2000).
The aim of combining all the women in the vertebral fracture arm (2027) with those in the clinical fracture arm with a femoral neck T score below -2.5 (1631) was to construct a larger cohort. This provided the opportunity to examine the effect of treatment on subgroups, the time course of these changes, and provide greater power to examine associations between variables. This was a true intention-to-treat analysis since all events after randomization were included.

The point estimates of the reduction in fracture risk (Table 3) were similar for those with and without a prevalent spine fracture and there was no significant heterogeneity in the relative risks between the two groups for any type of fracture. When the two groups were combined there was a significant reduction in all major fracture types varying from $27 \%-87 \%$ and the pattern and magnitude of change was similar whether osteoporosis was defined in terms of total hip or lumbar spine BMD. All fracture types showed some reduction of risk in the first year. They became significantly different from placebo by 12 months for clinical vertebral fractures (59\%), by 18 months for any clinical fracture (27\%) and hip fractures (63\%), by month 24 for non-vertebral fractures (26\%) and by month 30 for wrist fractures (30\%). These pivotal trials have been further analyzed and extended by additional studies to cover several practical aspects of the long term therapy for osteoporosis.

\section{Supporting studies Non-spine fractures}

Additional as well as supportive evidence as to the efficacy of alendronate was provided by the multinational Fosamax International Trial (FOSIT) (Pols et al 1999) where 1908 patients with a $\mathrm{T}$ score of -2 or less were treated for a year with $10 \mathrm{mg}$ daily or placebo. All participants were supplemented with $500 \mathrm{mg}$ calcium but no vitamin D. The primary end point was the change in BMD but non-spine

Table 3 Fracture risk in osteoporotic postmenopausal cohort*

\begin{tabular}{llll}
\hline Fracture type & $\begin{array}{l}\text { Vertebral Fx Arm } \\
\text { RR (95\% CI) }\end{array}$ & $\begin{array}{l}\text { Clinical Fx Arm } \\
\mathbf{( T < - 2 . 5 )} \\
\text { RR (95\% CI) }\end{array}$ & $\begin{array}{l}\text { Osteoporotic } \\
\text { cohort } \\
\mathbf{R R ~ ( 9 5 \% ~ C I ) ~}\end{array}$ \\
\hline Morphometric $\geq 1$ & & $0.51(0.31-0.84)$ & $0.52(0.42-0.66)$ \\
Morphometric $\geq 2$ & $0.53(0.41-0.68)$ & $0.40(0.08-1.95)$ & $0.13(0.07-0.25)$ \\
Clinical vertebral & $0.10(0.05-0.22)$ & $0.84(0.38-1.83)$ & $0.55(0.36-0.82)$ \\
Any clinical & $0.46(0.28-0.75)$ & $0.64(0.50-0.82)$ & $0.70(0.59-0.82)$ \\
Any non-vertebral & $0.74(0.59-0.92)$ & $0.65(0.50-0.83)$ & $0.73(0.61-0.87)$ \\
Hip & $0.81(0.64-1.03)$ & $0.44(0.18-0.97)$ & $0.47(0.26-0.79)$ \\
Wrist & $0.49(0.23-0.99)$ & $0.88(0.55-1.40)$ & $0.70(0.49-0.90)$ \\
\hline
\end{tabular}

Note: *Black et al 2000.

Abbreviations: $\mathrm{Cl}$, confidence interval; $\mathrm{Fx}$, fracture; $\mathrm{RR}$, relative risk. 
fractures were captured as an adverse event. Spine films were not performed at baseline and so the exact fracture risk status of the participants is not known, preventing direct comparison with FIT. At 12 months the gain in BMD with alendronate relative to placebo was $4.9 \%$ at lumbar spine, $2.4 \%$ at femoral neck, $3.6 \%$ at trochanter and $3.0 \%$ at total hip. These gains were similar to those seen in the dose response study (Liberman et al 1995) and were accompanied by a $52 \%$ decrease in bone specific alkaline phosphatase (BSAP) and a 74\% decrease in urine $\mathrm{N}$ terminal propeptide of type 1 collagen (NTx). At the end of a year, 37/958 (4.4\%) of the patients treated with placebo had experienced a nonspine fracture while the comparable figures for the alendronate group were 19/950 (2.4\%) representing a 47\% reduction in risk ( $\mathrm{RH} 0.53,95 \%$ CI $0.30-0.90$ ).

This study is important because of the rapidity of the protection against non-spine fractures, probably partly due to the use of the optimal $10 \mathrm{mg}$ dose of alendronate from the outset. It was also an important confirmation of the consistency of alendronate effect since the study was composed of a wide variety of racial groups and yet changes in BMD, bone turnover, and fracture protection were similar to those of earlier more homogeneous studies.

\section{High risk groups}

The vertebral fracture arm of FIT was re-analysed to explore the extent alendronate is able to reduce the fracture risk of the most vulnerable women (Ensrud et al 1997). In the study as a whole, the protective effect against both new vertebral and new clinical fractures was consistent across categories defined by age ( $<75$ years, $\geq 75$ years), median femoral neck $\operatorname{BMD}\left(<0.59 \mathrm{~g} / \mathrm{cm}^{2}, \geq 0.59 \mathrm{~g} / \mathrm{cm}^{2}\right)$, history of postmenopausal fracture (yes/no) and number of prevalent fractures (1 vertebral fracture, $\geq 2$ vertebral fractures). The practical implication of these findings is that they show that more fractures will be prevented by treating those women at highest risk defined by advanced age, low BMD, and multiple previous fractures.

\section{Prevention of multiple fractures}

The effect of alendronate on multiple symptomatic fractures was assessed from an intention to treat (ITT) analysis of the osteoporotic cohort of 3658 postmenopausal women in FIT (Levis et al 2002).

Most trials of osteoporosis censor (remove from further analysis) patients once they have had a fracture but there are obvious attractions to analyzing all events. Not only are multiple fractures of clinical importance but the greater the number of events available for analysis, then the impression of the effects of therapy are more complete.

However, because the first fracture increases the risk of subsequent events, the statistical analysis must adjust for the increase in risk of subsequent fractures due to the occurrence of the initial event. An addition attraction of this approach is that the number of events which contribute to the clinical outcome is increased, in this instance by $32 \%$, compared with trials which evaluate of the risk of first fracture after which the patient is censored from further analysis. There were 789 symptomatic fractures in the 4 year follow up with 86 multiple fractures in 1817 women treated with placebo and 51 fractures in 1841 women treated with alendronate. This equates to a relative risk reduction of multiple symptomatic fractures with alendronate of $42 \%$ (RR 0.58, 95\% CI 0.41-0.81). Comparable effects on multiple vertebral fractures were RR 0.16 (95\% CI 0.050.42 ) and on three or more fractures was RR 0.25 (95\% CI $0.10-0.55$ ) although the absolute number of the latter was very small. About half the patients with multiple symptomatic fractures had a prevalent vertebral fracture. The effect of alendronate became statistically significant at 6 months for multiple symptomatic vertebral fractures and at 12 months for multiple spine and non-spine symptomatic fractures. The reductions in risk occurred independent of age, prior morphometric or clinical vertebral fracture or the presence of a fall in the previous year.

It was also possible to examine the entire fracture experience of the osteoporotic cohort in terms of the first fracture in those with a low BMD as well as subsequent fractures in those who entered FIT with a prevalent fracture. Alendronate reduced the risk of a symptomatic fracture by 34\% (RR 0.66, 95\% CI 0.57-0.77) and symptomatic vertebral fractures by $63 \%$ (RR 0.37, 95\% CI 0.26-053) which is greater than the equivalent reductions of $30 \%$ and $45 \%$ based on first fracture only (Table 3). The results were the same when the whole cohort of 6459 women in FIT were subjected to the same statistical analysis although the benefits of alendronate were diluted by the women at low fracture risk because they were osteopenic without a prior fracture. The ability to prevent multiple fractures may alter the long term course of the disease since another analysis of FIT showed that the risk of future vertebral fractures increased with the number of prevalent fractures independent of age and BMD (Nevitt et al 1999). The ability of alendronate to attenuate this risk was not affected by the number or location of these prevalent fractures. 


\section{Predicting responses}

Since osteoporosis is a chronic condition requiring long term treatment, there is a real need to be able to monitor and predict response as well as to motivate patients to comply with treatment. Increased bone turnover is a well recognized predictor of fracture risk (Garnero et al 1996, 2000) and since substantial changes occur rapidly in response to potent inhibitors of bone turnover they have obvious application to the evaluation of therapy. Short term changes over 6 months in bone turnover markers, particularly urine NTx, were able to predict the gain in BMD at spine, hip, and total body at 24 months in elderly calcium and vitamin D supplemented women treated with either placebo or alendronate $5 \mathrm{mg}$ daily for the first year and then $10 \mathrm{mg}$ daily for the second year (Greenspan et al 1998). In this study, which was not powered for anti-fracture effect, the gains in BMD were similar to those seen in FIT. Only baseline NTx correlated with the gain in BMD and this was consistent with earlier reports (Bone et al 1997), but the change in all the markers, which comprised urine NTx and free deoxypyridinoline (Dpd), osteocalcin, and BSAP, at 6 months were predictive of gain in BMD. Only NTx and osteocalcin were predictive of gain at all measurement sites. Those with the largest decrease in NTx showed the biggest gains in BMD so that a $30 \%$ decrease in NTx predicted gains in BMD of $5.8 \%$ at lumbar spine, $4.1 \%$ at trochanter, and $2.8 \%$ at the total hip at 2.5 years. The indirect assumption is that the greater the gain in BMD, the greater the reduction in fracture risk (Wasnich and Miller 2000; Hochberg et al 2002).

More recently, data from FIT has been analyzed to establish whether there is an association between the change in bone turnover and the reduction in fracture risk (Bauer et al 2004). As would be expected, baseline hip BMD was associated with future fracture risk but baseline bone turnover markers were not correlated with any type of fracture outcome. The magnitude of the change in bone turnover over the first 12 months of alendronate therapy did predict the effect on spine, non-spine, and hip fractures. BSAP proved to be the most predictive and while serum $\mathrm{N}$ terminal propeptide of type 1 collagen (PINP) and serum C-terminal crosslinked telopeptide of type 1 collagen (CTx) were associated with a reduction in spine fractures, they only showed similar, but nonsignificant, associations with hip and non-spine fractures. In an age-adjusted logistic regression model, a 1 SD reduction in BSAP was associated with a $26 \%$ reduction in spine fractures (CI 13\%-37\%), an
$11 \%$ reduction in non-spine fractures (CI $0 \%-22 \%$ ) and a $39 \%$ reduction in hip fractures (CI 22\%-54\%). The relationship between \% change in bone turnover and reduction in fracture risk persisted after adjustment for change in spine BMD and did not differ when absolute changes in bone turnover were used. The results were also similar when analyses were restricted to women with a prevalent vertebral fracture or hip T score below -2.5 . The clinical utility of various cut points in marker change was also explored in order to predict fracture protection. A 30\% reduction in BSAP functions well in this respect and this is an area for potential development as the range of available markers expands and automated methodology improves precision. In contrast, the change in spine BMD did not predict fracture response and this was also true for hip BMD except for the ability to predict reduction in spine fractures.

A new vertebral fracture causes considerable disability with the main symptoms being back pain and kyphosis leading to reduced mobility. Treatment would be expected to reduce these complications by preventing new fractures and an analysis of the vertebral fracture arm of FIT showed that this appeared to be true. In this type of patient, back problems are very common, but alendronate was able to reduce the number of days that these osteoporotic women spent on bed rest by $32 \%$ or with 7 or more days of limited activity by $13 \%$. This seemed due to the ability of the drug to reduce the risk of new vertebral fractures (Nevitt et al 2000).

\section{Compliance and duration of treatment}

Treatment of a chronic condition such as osteoporosis, where symptoms are intermittent, is often accompanied by poor compliance and anything which might improve this situation is clearly an advantage. Bisphosphonates such as alendronate strongly adsorb to hydroxyapatite and remain active on the bone surface for several weeks during which time osteoclastic bone resorption will be continuously suppressed. In this way the effects of continuous daily administration can be achieved by intermittent dosing with the potential for improved compliance. In two studies (Schnitzer et al 2000; Rizzoli et al 2002) it was shown that administration of $70 \mathrm{mg}$ of alendronate once weekly and $10 \mathrm{mg}$ daily were equipotent in terms of the gain in BMD and reduction in bone turnover. In a study of 1258 postmenopausal women, with a BMD T score below -2.5 or a prior vertebral or hip fracture, who were randomized to 
alendronate $70 \mathrm{mg}$ once weekly $(\mathrm{n}=519), 35 \mathrm{mg}$ twice weekly ( $n=369)$ or $10 \mathrm{mg}$ daily $(n=370)$, the mean increase in $\mathrm{BMD}$ at the lumbar spine at 24 months was 6.8\%, 7.0\%, and $7.4 \%$ respectively. Similar equivalence of BMD gain was seen at the femoral neck, trochanter, and total body. Urine NTx , a marker of bone resorption, decreased by comparison with baseline by $73.4 \%, 71.3 \%$, and $74.9 \%$ in the weekly, twice weekly, and daily groups while the equivalent decreases in BSAP were 40.1\%, 41.7\%, and $43.1 \%$. None of these changes were significantly different between treatment groups. The reductions in NTx were similar across treatment groups independent of baseline $\mathrm{BMD}$, bone turnover, years since menopause, or age. Upper gastrointestinal adverse events occurred in $22.4 \%-23.8 \%$ of patients in the one year study and in $29.0 \%-30.0 \%$ in the two year study with no difference between treatment doses. There was no placebo group in these studies and so the incidence of upper gastrointestinal symptoms which might be expected in this type of population is unknown. Regulatory authorities require demonstration of bioequivalence for the new formulation to claim the same therapeutic efficacy on fracture protection as the formulation against which this has actually been tested. Subsequent experience has confirmed patient preference for weekly treatment and the improvement in long term compliance (McCombs et al 2004).

The prolonged adsorption of bisphosphonates to calcified bone surfaces makes possible the shift from daily to weekly therapy and this also influences the potential duration of treatment. The original 3 year pivotal phase III study (Liberman et al 1995) was extended out to ten years (Bone et al 2004) with an interim analysis at 7 years (Tonino et al 2000, 2001). At ten years, the effects on BMD and bone turnover of $5 \mathrm{mg}$ and $10 \mathrm{mg}$ daily continuous treatment with alendronate were compared with $20 \mathrm{mg}$ for 2 years and then 5mg daily for years 3-5 (discontinuation group). Randomization and blinding were maintained out to 10 years in the 247 women who remained in the study. Continuous treatment with $5 \mathrm{mg}$ and $10 \mathrm{mg}$ daily resulted in a sustained increase in BMD at all measurement sites (lumbar spine, femoral neck, trochanter, total hip, and total body). The major increase in BMD occurred in the first two years, but there was a steady increase as long as therapy was continued. These changes were consistent with filling in of the resorption space early in treatment (Heaney et al 1997) followed by more complete mineralization of the newly formed bone as it ages (Boivin et al 2000). Lumbar spine BMD increased by $13.7 \%$ after 10 years treatment with
$10 \mathrm{mg}$ daily but there were lesser gains at cortical sites, for example, at the total body, which increased by only $3 \%$. BMD began to increase when treatment was discontinued, but this was less marked at the spine compared with the hip. This may just reflect the effect of age-related posterior facet joint sclerosis offsetting losses of trabecular BMD from the vertebral body. Urine NTx and BSAP remained suppressed with continuous alendronate, but increased by about 25\% when treatment was discontinued although they remained below baseline for the 5 years of observation

The effect of these changes on fracture rate are difficult to assess since it is clearly unethical to have a placebo group of osteoporotic women who remain untreated for a decade. In this particular study, the effect of extended treatment was estimated by adjusting the fracture rate in the placebo group from the first three years of the study for the effects of increasing age and extrapolating out to ten years. The calculated and observed non-spine fracture rates were similar, which appeared reassuring and was taken to indicate that the effects of treatment were maintained. However it must be recognized that the absolute number of fractures in this residual cohort was small, which leads to an element of clinical and statistical uncertainty. Morphometric fractures were also recorded between years 6-10 in 228/247 women and showed no significant difference between groups (10 mg daily: 5\%, $5 \mathrm{mg}$ daily: 13.9\%, discontinuation; 6.6\%). There was also no difference in height loss between the groups which also suggests continuing benefit. These effects are consistent with long term retention of alendronate in bone which, although without effect when buried within bone, retains biological activity when uncovered by osteoclastic resorption. The practical implication of these observations is that after 5 years treatment, there is the opportunity to discuss the duration of treatment in the light of the published evidence. A patient who has had no fractures while on treatment may elect to discontinue treatment for a time providing some form of biochemical or densitometric monitoring is available to detect relapse. Patients who are anxious about continuing fractures may prefer to continue treatment and the 10 year study shows that this will maintain control of bone turnover and maintain gains in BMD.

Similar conclusions were derived from the long term extension of FIT (Ensrud et al 2004) where 1099 women previously treated with alendronate were randomly assigned to $5 \mathrm{mg}$ or $10 \mathrm{mg}$ of alendronate or placebo for a further 5 years. The women enrolled into this extension study were slightly younger, had a higher increase in total hip BMD, and were less likely to have had a new radiographic vertebral 
fracture compared with the women who were not enrolled (commonly because of a desire to continue on open label alendronate after completion of FIT). An interim analysis at 3 years showed that further therapy with $5 \mathrm{mg}-10 \mathrm{mg}$ of alendronate maintained BMD at the total hip, femoral neck, and trochanter, leading to a difference from placebo of about $2 \%$, while at the lumbar spine this difference was $2.5 \%$.The gains in BMD were slightly greater with the $10 \mathrm{mg}$ dose $(<1 \%)$ and reached statistical significance at the hip, but not at the spine. The rate of decline in BMD of the placebo group was similar to the background rate observed in the first 3 years of FIT but did not show "catch up" bone loss nor did this entirely erode the gains made by earlier treatment. Bone turnover remained stable and suppressed with both doses of alendronate. It increased by around $20 \%$ in those given placebo, but was still below baseline values after 3 years without therapy. These findings are consistent with a residual effect due to bisphosphonate retained within bone from earlier treatment, but fracture rates were not assessed at this stage and so the benefits from such prolonged treatment remain uncertain until the study is completed.

All these studies were generally reassuring about the long term use of alendronate.

A recent report of 9 cases who developed spontaneous non-spine fractures while on alendronate therapy and who showed delayed or absent fracture healing illustrates the need for clinicians to be alert for such issues (Odvina et al 2005). Iliac crest bone biopsies showed substantial suppression of bone formation with absent double tetracycline labeling, but less prominent reduction in biochemical markers of bone turnover. Bone resorption did not seem suppressed to the same degree but the changes were seen in both cancellous and cortical bone. All the patients were co-prescribed calcium and vitamin $\mathrm{D}$ as is current practice but three were also receiving estrogen and two prednisolone (one of whom also had hypoparathyroidism), which may have contributed to the picture. Fractures healed in most patients when alendronate was discontinued. The risk of this situation is unknown given the lack of a denominator and patients in the pivotal clinical trials described earlier do develop fractures while receiving alendronate and so a causal relation cannot be made. The onset of spontaneous nonspine fractures, particularly in unusual sites, should lead to a review of treatment and an assessment of the adequacy of fracture healing.

\section{Comparison with other therapies}

A major problem in the field of osteoporosis, and one which is unlikely to be resolved, is the direct comparison between drugs in terms of fracture end points. Even if there were clinically significant differences of $5 \%-10 \%$ between drugs, the size of such trials would be enormous, involving tens of thousands of patients (Kanis et al 2002), making the cost prohibitive. Comparisons can be made using surrogate markers such as BMD or bone turnover although uncertainty remains as to how differences might translate into fracture protection (Hochberg et al 2002; Delmas and Seeman 2004; Delmas et al 2004). Alendronate has been compared in this way with risedronate and raloxifene and hormone replacement therapy (HRT).

In a study of 1053 women with a low BMD (T score below -2) who were randomized between weekly alendronate $(70 \mathrm{mg}$ ) and risedronate $(35 \mathrm{mg}$ ) there were greater gains after 12 months treatment in BMD at all measurement sites with alendronate (Rosen et al 2005). These differences were statistically significant $(\mathrm{p}<0.001$ except femoral neck $\mathrm{p}<0.005$ ) but modest (trochanter: $1.4 \%$, lumbar spine $1.2 \%$, total hip: $1.0 \%$, femoral neck: $0.7 \%$ ). There were also significantly greater reductions $(\mathrm{p}<0.001)$ in bone turnover with alendronate (alendronate-risedronate difference: urine NTx 12.6\%, serum CTx 19.1\%, BSAP $12.5 \%$, PINP 15.9\%). This study was also interesting since it provided a direct comparison of the upper gut tolerability of these two bisphosphonates and found no significant difference. These effects on BMD, bone turnover, and adverse event profile were similar to an earlier study which compared weekly fasting alendronate with daily risedronate given 2 hours after a main meal, which was the licence at the time of the study for risedronate (Hosking et al 2003). It was not clear whether the lesser efficacy of risedronate was due to the daily between meal dosing or to an intrinsic characteristic of the compound. The findings of the later study are therefore more relevant since both agents are currently predominantly administered in the once weekly fasting formulation. It may be that where drugs have very similar characteristics, then small differences, such as better effects on BMD and bone turnover, have a substantial effect on choice and this may partly explain the dominance of alendronate in the treatment of postmenopausal osteoporosis.

Raloxifene, the selective estrogen receptor modulator (SERM) has a fundamentally different mode of action from the bisphosphonates and two recent studies have compared 
their effects on BMD and bone turnover (Luckey et al 2004; Sambrook et al 2004). Both trials recruited post menopausal women with low BMD (T score below -2.0) who were randomized to $70 \mathrm{mg}$ once weekly alendronate or $60 \mathrm{mg}$ daily raloxifene with appropriate placebo tablets to maintain blinding. Both studies showed similar results with greater increases in BMD and decreases in bone turnover with alendronate. In the multinational trial (Sambrook et al 2004) the mean gain in $\mathrm{BMD}$ with alendronate relative to raloxifene was $2.6 \%$ at the lumbar spine, $2.0 \%$ at trochanter, $1.3 \%$ at femoral neck, and $1.6 \%$ at the total hip. None of these differences were dependent on age or baseline BMD. The reductions in bone turnover measured as urine NTx and BSAP were approximately $40 \%$ greater with alendronate. In the US study (Luckey et al 2004), the mean gain in BMD with alendronate relative to raloxifene was $2.5 \%$ at the lumbar spine, $1.4 \%$ at trochanter, $0.3 \%$ at femoral neck, and $1.0 \%$ at the total hip. Only the differences at the lumbar spine, total hip, and trochanter reached statistical significance. Differences in the degree of bone turnover suppression in favor of alendronate were $45.5 \%$ for urine NTx and $24.8 \%$ for BSAP. In both studies withdrawals because of drug related or upper GI adverse events were similar between the two drugs although a greater proportion of patients reported vasomotor symptoms with raloxifene.

The use of alendronate and HRT in combination does give some insight into the relative effects of these two agents. In a group of postmenopausal women established on HRT for at least a year (mean duration of therapy 9.7 years) the addition of alendronate (10 mg daily) resulted in further increments in BMD and decrements in bone turnover (Lindsay et al 1999). The gains in BMD with the addition of alendronate were most marked at the lumbar spine $(+2.6 \%)$ and hip trochanter $(+2.2 \%)$, but did not reach statistical significance at the femoral neck (+0.9\%). These changes were less than those seen in treatment naïve patients due to the effects of the established HRT, but the study shows the greater potency of this dose of alendronate and suggests a clinical advantage of bisphosphonate in those women with very low BMD.

\section{Primary prevention}

Although current clinical practice has moved away from primary prevention for cost effectiveness reasons, there is clear evidence that alendronate is able to reduce the bone loss of recently postmenopausal women (Hosking et al 1998; Ravn et al 1999). The Early Postmenopausal Intervention
Cohort (EPIC) study compared placebo with estrogenprogestin and two doses of alendronate $(2.5 \mathrm{mg}$ and $5.0 \mathrm{mg}$ daily) in 1609 recently postmenopausal women aged 4559 years. Both doses of alendronate prevented the 4 year $2.9 \%$ decrease in BMD at the femoral neck seen in placebotreated women and produced a modest dose-related increase of $0.6 \%-1.4 \%$. Similar patterns of response were seen in the subgroup of women with osteopenia as well as in the total group at all measurement sites which included lumbar spine, trochanter, total hip, total body, and distal one third radius. The gain in BMD was greatest in women who were further away from the onset of the menopause as is to be expected from the observation that bone loss is most rapid in the early menopausal years (Bjarnasson et al 2002). Women treated with estradiol-norethisterone gained 3.7\% at the femoral neck while those given estrogenmedroxyprogesterone acetate gained $1.8 \%$. Both hormonal treatments were also more effective than these low doses of alendronate at the lumbar spine, total body, and forearm. The switch from alendronate to placebo after 2 years in a subset of women resulted in a resumption of bone loss at a comparable rate seen in placebo-treated women at the beginning of the study. Even so those women treated with $5 \mathrm{mg}$ of alendronate were still above baseline at the hip and spine with a more marked benefit compared with the continuously treated placebo group. Bone turnover (NTx, osteocalcin, and BSAP) decreased into the premenopausal reference range with patterns of response consistent with the changes in BMD. The study was not powered to show an effect on fractures, which have a low frequency in this early postmenopausal population (Doherty et al 2001).

Important differences between alendronate and hormone treatment emerged when therapy was discontinued after 4 years (McClung et al 2004; Wasnich et al 2004). Women treated continuously with alendronate for 6 years maintained BMD at all measurement sites while those in whom treatment was discontinued after four years lost bone at a similar rate to placebo. In contrast the women treated with hormonal therapy for 4 years had significantly greater mean BMD losses at all sites during the 2 years after stopping treatment than did those who had received alendronate $5 \mathrm{mg}$ for 4 years. BMD changes during the two years off hormone or bisphosphonate treatment were, respectively: lumbar spine (-7.69\% and $-2.42 \%)$, total hip (-5.16\% and $-1.09 \%)$, femoral neck ( $-5.80 \%$ and $-0.57 \%)$, trochanter $(-7.22 \%$ and $-0.31 \%)$, forearm $(-2.54 \%$ and $-0.84 \%)$ and total body $(-5.58 \%$ and $-2.90 \%)$. The pattern of offset of treatment effect on bone turnover was similar to that of BMD. These 
changes reflect the residual effect of bisphosphonate retained in bone but the rapid loss of hormonal occupancy of receptors.

\section{Alendronate in the management of male osteoporosis}

Osteoporosis has up to recently been considered as a disease that mainly affects women, and therefore most studies on the treatment of osteoporosis has focused on female osteoporosis. Age-related osteoporosis in men usually only manifests at a later stage than in women (Usually $>70$ years of age). Men that present with osteoporosis at a younger age often have an underlying illness that increases bone loss or decreases bone formation such as hypogonadism, alcoholism, glucocorticoid treatment, anticonvulsant therapy, and rheumatoid arthritis (Orwoll and Klein 2001). Men are less likely to fall than women and have a lifetime risk of osteoporotic fracture that is around one third of that in women. The lifetime risk of a hip, spine, or forearm fracture is $40 \%$ in white women and only $13 \%$ in white men (Melton et al 1992, 1998). Although the major trials for treatment of osteoporosis have mainly focused on osteoporosis in females there have been several randomized, controlled clinical studies that investigated the treatment of osteoporosis in men.

Orwoll et al (2000) showed in a randomized controlled study that alendronate given with calcium and vitamin D was effective in the treatment of male osteoporosis. This double blind, randomized study over a period of 2 years compared the effect of daily alendronate $10 \mathrm{mg}$ or placebo on BMD in 241 men with osteoporosis. Thirty-seven of these men were hypogonadal as evidenced by low free testosterone concentrations with the rest having normal testosterone concentrations. The age of the enrolled subjects ranged between 31 and 87 years with a mean age of 63 years. Subjects were enrolled if they had a femoral neck Tscore of -2.0 SD or less and a lumbar spine T-score of -1.0 $\mathrm{SD}$ or less below the mean for a young healthy male. Male subjects were also enrolled if they had a femoral neck Tscore of at least $1 \mathrm{SD}$ below the young adult mean and at least one vertebral deformity or a history of osteoporotic fracture. All men with a secondary cause for osteoporosis except hypogonadism were excluded from the trial.

The alendronate treatment group showed a mean increase in BMD of $7.1 \% \pm 0.3 \%$ at the lumbar spine, $2.5 \% \pm 0.4 \%$ at the femoral neck compared with an increase in the control group of $1.8 \% \pm 0.5 \%$ at the lumbar spine $(\mathrm{p}<0.001$ for comparison with baseline) and a reduction of $-0.1 \% \pm 0.5 \%$ at the femur neck (insignificant difference). The increase in the BMD in the alendronate group was significantly greater than in the placebo group at all measurement sites $(\mathrm{p}<0.001)$. There were similar increases in BMD in the hypogonadal and the eugonadal men treated with alendronate. When quantitative measurements were done for vertebral fractures, the vertical fracture incidence was significantly lower in the alendronate group than in the placebo group $(0.8 \%$ vs $7.1 \%, \mathrm{p}=0.02$ ). There was no significant difference in the rate of non-vertebral fractures between the placebo and alendronate-treated groups ( $5.3 \%$ vs $4.1 \%$, respectively). The results conclusively show alendronate treatment is beneficial in male patients with osteoporosis and also that gonadal status does not influence the effect of alendronate on BMD.

A further prospective study done by Ringe et al (2001) confirmed the benefits of alendronate in the treatment of male osteoporosis. This single centre, randomized prospective 2-year study enrolled 134 men with established primary osteoporosis. Any patient with hypogonadism or other causes of secondary osteoporosis were excluded from this study. No patient that was involved with this study had any previous exposure to bisphosphonate treatment. Subjects were randomized to receive either alendronate (10 mg daily) or 1-alphacalcidol (1 $\mu$ g daily) and all subjects received an additional $500 \mathrm{mg}$ per day of calcium supplementation. None of the patients received any other vitamin D replacement or other supplements.

Patients were seen at baseline and followed at 6, 12, 18, and 24 months. Each visit included a physical examination with height measurements. BMD of the lumbar spine and femoral neck were done at baseline and were again repeated at each follow up visit. X-rays of the spine were obtained at baseline and this was repeated annually. Primary end points of the study were to measure the change in lumbar spine and femoral neck BMD over time. Secondary end points included vertebral and non-vertebral fractures, and stature.

Lumbar spine density in the alendronate group increased by $10.1 \%$ at year 2 , and at the femur neck BMD increased by $5.2 \%$. The alphacalcidol group showed an increased BMD of $2.8 \%$ at the lumbar spine and $2.2 \%$ at the femur neck. P-values for the differences in the femur neck and lumbar spine BMD in the two treatment groups were 0.001 and 0.009 respectively. The study showed that the total number of new vertebral fractures was significantly reduced in the alendronate treatment group (7.3\% in alendronate group vs $18.2 \%$ in alphacalcidol group, $\mathrm{p}=0.071$ ). Although 
the 1-alphacalcidol group showed a significant increase from baseline in the lumbar spine BMD, these patients did not have the same benefit with regards to new vertebral fractures. There was no significant difference in the incidence of non-vertebral fractures $(8.7 \%$ in alendronate group and $12.1 \%$ in the alphacalcidol group).

The results of this study were consistent with those reported by Orwoll et al (2000) Both these studies confirmed that men with established osteoporosis benefit from treatment with alendronate and have an increased lumbar spine and femoral neck BMD and also showed that alendronate is generally well tolerated when used in a daily format. The recommended dosage of alendronate for the treatment of osteoporosis in men is $70 \mathrm{mg}$ orally once weekly or $10 \mathrm{mg}$ orally once daily.

\section{Alendronate and parathyroid hormone in the treatment of osteoporosis}

Most therapies that are currently used for the management of osteoporosis act mainly to inhibit bone resorption and therefore reduce bone remodeling. Teriparatide, recombinant human parathyroid hormone (PTH 1-34), was the first anabolic bone agent for the treatment of osteoporosis licensed for use in the US and the UK. It increases new bone formation by increasing osteoblast differentiation, osteoblast function, and survival. Teriparatide is recommended for patients with severe osteoporosis and can only be administered by once-daily injection in the thigh or abdomen. The recommended dose is 20 mcg per day. The maximum duration of use that is recommended is 18 months. Parathyroid hormone is known to increase both bone formation and bone absorption and treatment of osteoporosis with PTH causes a marked increase in vertebral BMD. However, this effect is rapidly reversed when the treatment is stopped.

It was uncertain whether a combination of alendronate and PTH would lead to an enhanced effect on bone and also whether PTH treatment followed by alendronate would preserve or increase bone density in patients previously treated with PTH. The use of PTH in combination with alendronate and also PTH followed by alendronate has been investigated in several studies (Rittmaster et al 2000; Neer et al 2001; Black et al 2003; Finkelstein et al 2003).

Finkelstein et al (2003) randomized 83 men between the ages of 46-85 known to have low BMD into three treatment groups. The men received alendronate $10 \mathrm{mg}$ daily
(28 men), PTH $40 \mu$ g subcutaneously (27 men), or both (28 men). The subjects on alendronate only or combination of PTH and alendronate were started on alendronate $10 \mathrm{mg}$ per day for a total of 30 months. PTH (1-84) was begun 6 months into the study and continued for a further 2 years. BMD of the lumbar spine, proximal femur, radial shaft, and total body was measured every 6 months using a dual energy $\mathrm{x}$-ray absorbed geometry and densitometry scanner. For the radial shaft two measurements were obtained at each visit, and the mean of the two values were used in analysis. BMD of the lumbar spine in the posterior anterior and lateral projections and the proximal femur as well as the distal one third of the radial shaft was measured. The study also included measuring trabecular BMD of the lumbar spine using computer tomography.

The results of the study confirmed what was already known about the administration of alendronate as a single agent. On the posterior-anterior spine, there was a mean increase in BMD of $7.9 \%$ and of the lateral spine $11.1 \%$. Femur neck density increased by $3.2 \%$ with a total hip density increasing by $4.8 \%$. The PTH group had a clinically significant mean percentage increase in BMD of 18.1\%, $25.8 \%, 9.7 \%$, and $6.4 \%$ at the posterior-anterior spine, lateral spine, femoral neck, and total hip respectively. The theoretical benefit from a combination of an anabolic bone agent (teriparatide) and an inhibitor of bone resorption (alendronate) did not seem to have any advantage. In the combination therapy group, the mean increase in BMD was almost midway between the alendronate-only group at the lower end of the scale and the PTH group only at the higher end of the scale. The mean percent increase in BMD in the combination group was $14.8 \%, 18.0 \%, 6.2 \%$, and $5.3 \%$ at the posterior-anterior spine, lateral spine, femoral neck, and total hip respectively. The lumbar spine effects of the combination of alendronate and teriparatide were no better than either agent alone, where as the total hip combination was no different from that of the alendronate alone. The study suggested that alendronate significantly impairs the anabolic effect of teriparatide, especially if patients have been pre-treated with alendronate.

Black et al (2003) conducted a similar study in 238 women with a T-score of less than -2.5 for BMD at the femoral neck, total hip, or spine. They also included women with a T-score of less than -2.0 at one of the above sites, but who also had a further risk factor (age $\geq 65$ years, a history of postmenopausal fracture and a maternal history of hip fracture. The women were randomly assigned to one of three treatment groups for a period of one year (the study was 
later extended to 2 years - see below). Women were assigned to take PTH plus placebo $(n=119)$, PTH plus alendronate $(n=59)$, and alendronate plus placebo $(n=60)$. All the subjects received a daily dose of calcium and vitamin $\mathrm{D}$ supplement. BMD was measured at the femoral neck and total hip regions, the postero-anterior lumbar spine (L1 to L4), and also at the distal one third of the radial shaft. Measurements were done at baseline and again at 12 months.

The results showed that BMD increased in all the women receiving treatment and there were no significant difference between the PTH monotherapy and the PTH-alendronate combination group. The areal BMD of the lumbar spine increased by $6.3 \%$ in the PTH monotherapy group and by $6.1 \%$ in the PTH-alendronate combination group. The femoral neck and total hip BMD remained essentially unchanged in the PTH monotherapy group but there was a significantly greater increase in total hip BMD in the PTHalendronate combination group (0.3\% vs. 1.9\%). There was also an increase of the femoral neck and total hip BMD in the alendronate monotherapy group. The distal radius BMD decreased significantly in the PTH group (-3.4\%), but results showed that treatment with alendronate might decrease the rate of bone loss. BMD loss in PTH-alendronate group was $-1.1 \%$ with similar loss in the alendronate monotherapy group.

One of the great strengths of PTH is the significant increase in vertebral BMD, but PTH is currently not licensed for long-term maintenance treatment of osteoporosis. An attractive alternative theory emerged whereby the benefit of PTH on bone remodeling could be maintained after withdrawal of PTH by treating a patient with an antiresorptive agent such as alendronate. Rittmaster et al (2000) conducted a study to determine whether alendronate would prevent bone loss or even enhance BMD in patients previously treated with PTH. Postmenopausal women $(n=66)$ with a diagnosis of osteoporosis were treated for 1 year with either recombinant PTH (1-84) using the doses $50 \mathrm{mcg}$, $75 \mathrm{mcg}$, or $100 \mathrm{mcg}$ or were given placebo. Note that the dose of PTH (1-84) differs from that of teriparatide (PTH 1-34). All subjects were then were given a daily dose of alendronate $10 \mathrm{mg}$ daily for an additional year. Femoral neck, lumbar spine, and whole body BMD was measured. During the first year of the study, changes in BMD (mean \pm $\mathrm{SD}$ ) in women receiving PTH (all doses combined) were $7.1 \% \pm 5.6 \%$ (spine), $0.3 \% \pm 6.2 \%$ (femoral neck), and $-2.3 \% \pm 3.3 \%$ (total body). After withdrawal of PTH and starting on alendronate for the next year in the women who had received $\mathrm{PTH}$, the mean changes in BMD were
$13.4 \% \pm 6.4 \%$ (spine), $4.4 \% \pm 7.2 \%$ (femoral neck), and $2.6 \% \pm 3.1 \%$ (whole body). This study showed that sequential treatment of osteoporotic patients with PTH followed by alendronate results in a further increase in vertebral BMD that is significantly higher than BMD gained with PTH only and also with simultaneous alendronate and PTH.

In a recent extension of the study by Black et al (2003), the investigators reported on the question of whether PTH treatment should be followed by antiresorptive therapy such as alendronate (Black et al 2005). Women who had received PTH (1-84) monotherapy $100 \mu$ g per day, in the first year were then randomized to receive either alendronate $(n=59)$ or placebo $(n=60)$ for an additional year. The study also extended the treatment of subjects who had received a combination of alendronate and PTH in year one, to continue these patients with alendronate only in the second year of the study. Those subjects who had received alendronate monotherapy in the first year, continued with the same therapy during year two. Changes in BMD at the hip and spine was assessed as before through the use of DEXA scanning and quantitative computer tomography (CT).

The results of the study showed that in the women who received $\mathrm{PTH}$ for 12 months followed by alendronate $10 \mathrm{mg}$ daily for a further 12 months, there was a significant additional increase in BMD at the spine $(4.9 \%, \mathrm{p}<0.001)$ and also a significant increase in BMD at the hip (3.6\% $\mathrm{p}<0.001$ ).

The women, who had received PTH for the first 12 months of this study and was followed by placebo treatment for a further 12 months, had a significant decrease in BMD at the spine $(-1.7 \%, \mathrm{p}=0.002)$ with no significant change in bone density at the hip or radius. The difference in BMD gain in year two between PTH-alendronate group and bone loss in the PTH-placebo group was shown to be significant at the spine and the total hip.

At the time of the study, teriparatide had only been recently approved for use in osteoporosis and it was not clear whether there would be any benefit in combining teriparatide with an antiresorptive drug such as alendronate. The trial was designed to investigate what effect different combinations would have on BMD at the spine and femur neck. It was shown that using PTH (1-84) in combination with alendronate did not provide any clear benefit over PTH only. This study confirmed the findings of previous reports that alendronate therapy following PTH leads to further increase in BMD and is likely to protect against increased 
bone resorption following withdrawal of PTH (Lindsay et al 1997; Rittmaster et al 2000; Kurland et al 2004; Lindsay et al 2004).

\section{Alendronate in corticosteroid- induced osteoporosis}

Glucocorticoids are widely used to treat a number of major illnesses such as chronic obstructive pulmonary disease, inflammatory bowel disease, and other inflammatory diseases such as rheumatoid arthritis and polymyalgia rheumatica. Corticosteroids are also commonly used for immunosuppression in transplant recipients and also in the management of certain hematological malignancies (Walsh et al 1996).

The benefit of glucocorticoid treatment has to be weighed up against the numerous side effects that the users suffer. One of the long-term side effects of glucocorticoid use is the development of osteoporosis. The administration of oral glucocorticoids is associated with a significant increase in fracture risk at the hip and spine. The greatest increase in osteoporosis risk is associated with intake of high doses of glucocorticoids, however an increased risk is seen with daily administration of prednisolone at doses lower than $7.5 \mathrm{mg}$. The risk for osteoporotic fractures also increases after initiating glucocorticoid treatment, but this declines soon after stopping treatment (Van Staa et al 2000, 2002).

Corticosteroid-induced osteoporosis results from the effect of corticosteroids on bone and calcium homeostasis. Direct effects of steroids include the suppression of intestinal calcium absorption with increases to urinary, calcium, and phosphate loss. Steroids lead to a suppression of osteoblast precursor formation with increased apoptosis of mature osteoblasts. Glucocorticoid treatment inhibits synthesis and secretion of bone matrix proteins, growth factors, and cytokines and reduces androgen secretion. Furthermore it leads to an increase in osteoclast formation leading to net a reduction in bone formation and increased resorption. It is thought that a reduction of calcium and phosphate absorption from the gut and increased renal losses, may lead to lowgrade secondary hyperparathyroidism with further increased osteoclast activity (Cosman et al 1994; Patschan et al 2001; Weinstein 2001; Canalis et al 2004)

Several studies investigated the efficiency of alendronate for the prevention and treatment of glucocorticoid-induced osteoporosis. Gonnelli et al (1997) demonstrated the effectiveness of alendronate in preventing osteoporosis in patients with sarcoid treated with glucocorticoid drugs.
Forty-three patients with sarcoid (17 men and 26 premenopausal women) were included in the study. Thirteen of these patients did not need any glucocorticoid treatment and served as controls. The remaining 30 patients that needed glucocorticoid therapy were randomized to receive either placebo or alendronate $5 \mathrm{mg}$ per day. BMD was measured at the ultra-distal radius and biochemical markers of bone turnover were measured at baseline and after 6 and 12 months of glucocorticoid therapy. The glucocorticoid dose was not significantly different between the placebo and alendronate group. The alendronate-treated group's BMD increased by $0.8 \%$ and the BMD in the placebo group decreased by $4.5 \%(\mathrm{p}<0.01)$ There was a significant decrease in markers of bone formation in all patients on glucocorticoids, however, the alendronate-treated group had a reduction in the biochemical markers of bone resorption compared with the placebo group. This relatively small study confirmed that alendronate is effective in preventing glucocorticoid-induced bone loss.

Saag et al (1998) carried out two 48 week randomized, placebo-controlled studies on the use of alendronate in male and female patients (17-83 years of age). Investigators enrolled 477 men and women with underlying pulmonary, rheumatological, dermatological, gastrointestinal, or other diseases that required long-term oral glucocorticoid treatment at a dose of at least $7.5 \mathrm{mg}$ per day. The first multicenter study enrolled 232 patients in the US and the second multi-center study enrolled 328 patients in 15 other countries. Patients were randomly signed to receive $2.5 \mathrm{mg}$, $5 \mathrm{mg}$, or $10 \mathrm{mg}$ of oral alendronate or a matching placebo. All patients in both the studies were treated for a minimal of 48 weeks. Subjects received daily supplements of calcium and vitamin D. Subjects were stratified for the duration of the glucocorticoid use in three groups (<4 months, 4-12 months, and $>12$ months). Mean daily dose of glucocorticoid use was $10 \mathrm{mg}$ of prednisolone (or equivalent). BMD of the lumbar spine and hip as well as the total body BMD was measured at baseline and again at 12, 24, and 48 weeks by dual energy X-ray absorptiometry (DEXA scan). At the same time serum and urine samples were obtained for baseline measurement of markers of bone turnover (serum BSAP and urinary excretion of cross-linked n-terminal telopeptides of type 1 collagen).

The investigators reported that alendronate significantly increased lumbar spine and hip BMD in the $5 \mathrm{mg}$ and $10 \mathrm{mg}$ alendronate groups. The lumbar spine BMD was increased by $2.1 \%$ and $2.9 \%$ with femur neck BMD increased by $1.2 \%$ and $1.0 \%$ in the $5 \mathrm{mg}$ and $10 \mathrm{mg}$ alendronate groups 
respectively. The placebo group had minimal bone loss at the lumbar spine of $-0.4 \%$ and a reduction of BMD at the femur neck of $-1.2 \%$. There was a reduction in markers of bone resorption and formation in both the $5 \mathrm{mg}$ and $10 \mathrm{mg}$ alendronate groups. This study showed that alendronate significantly increases BMD in patients with glucocorticoid-induced osteoporosis and patients who are at high risk of developing osteoporosis on glucocorticoid treatment. The benefit of alendronate therapy was seen in men, pre-menopausal women, and post-menopausal women whether or not they were receiving HRT at the time. The vertebral fracture rate was reduced by approximately $50 \%$ in the alendronate treatment group although this was only statistically significant among the post-menopausal women; the reason for this is probably because the younger patients were at lower risk of vertebral fractures anyway.

A 12-month extension of the above study (Saag et al 1998) was reported in 2001 (Adachi et al 2001). This showed that all patients receiving alendronate had a further increase in lumbar spine and trochanter BMD in the 12-month extension period, while maintaining the BMD at the femoral neck. Patients receiving placebo continued to lose bone in the extension period at all sites. Alendronate therapy (5mg, $10 \mathrm{mg}$, or $2.5 / 10 \mathrm{mg}$ daily) to a total of two years increased lumbar spine BMD by between $2.8 \%$ and $3.9 \%$, a significant improvement compared with placebo ( $0.8 \%$ decrease). Incidents of vertebral fractures were reduced in the alendronate group versus the placebo group $(0.7 \%$ vs $6.8 \%$ respectively).

According to the above studies, alendronate has been shown to be effective at increasing BMD of the spine and total hip and also reduces vertebral fractures in patients on long-term glucocorticoid therapy. Following on from these studies, routine BMD testing and early treatment with bisphosphonates have been recommended by several groups such as the Royal College of Physicians of London and the American College of Rheumatology Ad Hoc Committee on Glucocorticoid-Induced Osteoporosis.

\section{References}

Adachi JD, Saag KG, Delmas PD, et al. 2001. Two-year effects of alendronate on bone mineral density and vertebral fractures in patients receiving glucocorticoids: a randomised, double-blind, placebo-controlled extension trial. Arthritis Rheum, 44:202-11.

Bauer DC, Black DM, Garnero P, et al. 2004. Changes in bone turnover and hip, non spine, and vertebral fracture in alendronate-treated women: The Fracture Intervention Trial. J Bone Miner Res, 19:12508.
Bjarnasson NH, Alexandersen P, Christiansen C. 2002. Number of years since menopause: spontaneous bone loss is dependent but response to hormone replacement therapy is independent. Bone, 30: 637-42.

Black DM, Cummings SR, Karpf DB, et al.1996. Randomised trial of effect of alendronate on risk of fracture in women with existing vertebral fractures. Lancet, 348:1535-41.

Black DM, Thompson DE, Bauer DC, et al. 2000. Fracture risk reduction with alendronate in women with osteoporosis: The fracture intervention trial. J Clin Endocrinol Metab, 85:4118-24.

Black DM, Greenspan SL, Ensrud KE, et al. 2003. The effects of parathyroid hormone and alendronate alone or in combination in postmenopausal osteoporosis. N Engl J Med, 349:1207-15.

Black DM, Bilezikian JP, Ensrud KE, et al. 2005. One year of alendronate after one year of parathyroid hormone (1-84) for osteoporosis. $N$ Engl J Med, 353:555-65.

Boivin GY, Chavassieux PM, Santora AC, et al. 2000. Alendronate increases bone strength by increasing the mean degree of mineralization of bone tissue in osteoporotic women. Bone, 27:68794.

Bone HG, Downs RW Jr, Tucci JR, et al. 1997. Dose-response relationships for alendronate treatment in osteoporotic elderly women. Alendronate Elderly Osteoporosis Study Centres. J Clin Endocrinol Metab, 82:26574.

Bone HG, Hosking D, Devogelaer J-P, et al. 2004. Ten years experience with alendronate for osteoporosis in postmenopausal women. $N$ Engl J Med, 350:1189-99.

Canalis E, Bilezikian JP, Angeli A, et al. 2004. Perspectives on glucocorticoid-induced osteoporosis. Bone, 34:593-98.

Cosman F, Nieves J, Herbert J, et al. 1994. High-dose glucocorticoids in multiple sclerosis patients exert direct effects on the kidney and skeleton. J Bone Miner Res, 9:1097-105.

Cummings SR, Black DM, Thompson DE, et al. 1998. Effect of alendronate on risk of fracture in women with low bone density but without vertebral fractures. JAMA, 280:2077-82.

Delmas PD, Li Z, Cooper C. 2004. Relationship between changes in bone mineral density and fracture risk reduction with anti-resorptive drugs: some issues with meta-analyses. J Bone Miner Res, 19:330-7.

Delmas PD, Seeman E. 2004. Changes in bone mineral density explain little of the reductionin vertebral or nonvertebral fracture risk with anti-resorptive therapy. Bone, 34:599-04.

Doherty D, Sanders K, Kotowicz M et al. 2001. Lifetime and five-year age-specific risks of first and subsequent osteoporotic fractures in postmenopausal women. Osteoporos Int, 12:16-23.

Ensrud DE, Black DM, Palermo L, et al 1997. Treatment with alendronate prevents fractures in women at highest risk. Results from the Fracture Intervention Trial. Arch Intern Med, 157:2617-24.

Ensrud KE, Barrett-Connor EL, Schwartz A, et al. 2004. Randomized trial effects of alendronate continuation versus discontinuation in women with low BMD: results from the Fracture Intervention Trial Long-term Extension. J Bone Miner Res, 19:1259-69.

Finkelstein JS, Hayes A, Hunslemann JL, et al. 2003. The effects of parathyroid hormone, alendronate, or both in men with osteoporosis. N Engl J Med, 349:1216-26.

Garnero P, Hausherr E, Chapuy MC, et al. 1996. Markers of bone resorption predict hip fractures in elderly women:The EPIDOS prospective study. J Bone Miner Res, 11:1531-8.

Garnero P, Sornay-Rendu E, Claustrat B, et al. 2000. Biochemical markers of bone turnover, endogenous hormones and the risk of fractures in postmenopausal women: The OFLEY study. J Bone Miner Res, 15:1526-36

Gonnelli S, Rottoli P, Cepollaro C, et al. 1997. Prevention of corticosteroidinduced osteoporosis with alendronate in sarcoid patients. Calcif Tissue Int, 61:382-5.

Greenspan SL, Parker RA, Ferguson L, et al. 1998. Early changes in biochemical markers of bone turnover predict the long term response to alendronate in representative elderly women: A randomized clinical trial. J Bone Miner Res, 13:1431-8. 
Heaney RP, Yates AJ, Santora AC. 1997. Bisphosphonate effects and the bone remodelling transient. J Bone Miner Res, 12:1143-51.

Hochberg MC, Greenspan S, Wasnich RD, et al. 2002. Changes in bone density and turnover explain the reductions in incidence of nonvertebral fractures that occur during treatment with antiresorptive agents. J Clin Endocrinol Metab, 87:1586-92.

Hosking D, Adami S, Felsenberg D, et al. 2003. Comparison of change in bone resorption and bone mineral density with once-weekly alendronate and daily risedronate: a randomized placebo controlled study. Curr Med Res Opin, 19:383-94.

Hosking D, Chilvers CED, Christiansen C, et al. 1998. Prevention of bone loss with alendronate in postmenopausal women under 60 years of age. New Engl J Med, 338:485-92.

Kanis JA, Oden A, Johnell O, et al. 2002. Uncertain future of trials in osteoporosis. Osteoporos Int, 13:443-9.

Klozbuecher CM, Ross PD, Landsman PB, et al. 2000. Patients with prior fractures have an increased risk of future fractures: a summary of the literature and statistical synthesis. J Bone Miner Res, 15:721-39.

Kurland ES, Heller SL, Diamond B, et al. 2004. The importance of bisphosphonate therapy in maintaining bone mass in men after therapy with Teriparatide (human parathyroid hormone 1-34). Osteoporos Int, 15:992-7.

Levis S, Quandt SA, Thompson D, et al. 2002. Alendronate reduces the risk of multiple symptomatic fractures: results from the Fracture Intervention Trial. J Am Geriatr Soc, 50:409-15.

Liberman UA, Weiss SR, Broll J, et al. 1995. Effect of alendronate on bone mineral density and the incidence of fractures in postmenopausal osteoporosis. $N$ Engl J Med, 333:1437-43.

Lindsay R, Nieves J, Formica C, et al. 1997. Randomised controlled study of effect of Parathyroid Hormone on vertebral bone mass and fracture incidents amongst post menopausal women on oestrogen with osteoporosis. Lancet, 350:550-5.

Lindsay R, Cosman F, Lobo RA, et al. 1999. Addition of alendronate to ongoing hormone replacement therapy in the treatment of osteoporosis: a randomized, controlled clinical trial. J Clin Endocrinol Metab, 84:3076-81.

Lindsay R, Scheele WH, Neer R, et al. 2004. Sustained vertebral fracture risk reduction after withdrawal of Teriparatide in postmenopausal women with osteoporosis. Arch Intern Med, 164:2024-30.

Looker AC, Johnston CC Jr, Wahner HW, et al. 1995. Prevalence of low femoral bone density in older U.S women from NHANES III. $J$ Bone Miner Res, 10:796-02.

Luckey M, Kagan R, Greenspan S, et al. 2004. Once-weekly alendronate $70 \mathrm{mg}$ and raloxifene $60 \mathrm{mg}$ daily in the treatment of postmenopausal osteoporosis. Menopause, 11:405-15.

Mc Combs JS, Thiebaud P, McLaughlin-Miley C, et al. 2004. Compliance with drug therapies for the treatment and prevention of osteoporosis. Maturitas, 48:271-87.

McClung MR, Wasnich RD, Hosking DJ, et al. 2004. Prevention of postmenopausal bone loss: six year results from the Early Postmenopausal Intervention Cohort study. J Clin Endocrinol Metab, 89:4879-85.

Melton III LJ, Chrischilles EA, Cooper C, et al. 1992. Perspective: How many women have osteoporosis? J Bone Miner Res, 7:1005-10.

Melton III LJ, Atkinson EJ, O’Connor MK, et al. 1998. Bone density and fracture risk in men. $J$ Bone Miner Res, 13:1915-23.

Neer RM, Arnaud CD, Zanchetta JR, et al. 2001. Effect of parathyroid hormone (1-34) on fractures and bone mineral density in postmenopausal women with osteoporosis. $N$ Engl J Med, 344:1434-41.

Nevitt MC, Ross PD, Palermo L, et al. 1999. Association of prevalent vertebral fractures, bone density, and alendronate treatment with incident vertebral fractures: effect of number and spinal location of fractures. Bone, 25:613-19.

Nevitt MC, Thompson DE, Black DM, et al. 2000. Effect of alendronate on limited-activity days and bed-disability days caused by back pain in postmenopausal women with existing fractures. Arch Intern Med, 160:77-85.
Odvina CV, Zerwekh JE, Rao DS, et al. 2005. Severely suppressed bone turnover: a potential complication of alendronate therapy. J Clin Endocrinol Metab, 90:1294-301.

Orwoll E, Ettingham M, Vice S, et al. 2000. Alendronate for the treatment of osteoporosis in men. $N$ Engl J Med, 343:604-10.

Orwoll ES, Klein R. 2001. Osteoporosis in men. Epidemiology, pathophysiology, and clinical characterization. In: Marcus R, Feldman D, Kelsey J (eds). Osteoporosis. 2nd ed. San Diego: Acad Pr. p 103-49.

Patschan D, Loddenkemper K, Buttgereit F. 2001. Molecular mechanisms of glucocorticoid-induced osteoporosis. Bone, 29:498-505.

Pols HAP, Felsenberg D, Hanley DA, et al. 1999. Multinational, placebo controlled, randomized trial of the effects of alendronate on bone density and fracture risk in postmenopausal women with low bone mass: results of the FOSIT study. Osteoporos Int, 9:461-8.

Ravn P, Bidstrup M, Wasnich RD, et al. 1999. Alendronate and estrogenprogestin in the long-term prevention of bone loss: Four year results from the Early Postmenopausal Intervention Cohort study. Ann Intern Med, 131: 935-42.

Ringe JD, Faber H, Dorst A. 2001. Alendronate treatment of established primary steoporosis in men: results of a 2-year prospective study. $J$ Clin Endocrinol Metab, 86:5252-55.

Rittmaster RS, Bolognese M, Ettinger MP et al. 2000. Enhancement of bone mass in osteoporotic women with parathyroid hormone followed by alendronate. J Clin Endocrinol Metab, 85:2129-34.

Rizzoli R, Greenspan SL, Bone HG, et al. 2002. Two year results of once weekly administration of alendronate $70 \mathrm{mg}$ for the treatment of postmenopausal osteoporosis. J Bone Miner Res, 17:1988-96.

Rosen CJ, Hochberg MC, Bonnick SL, et al. 2005. Treatment with onceweekly alendronate $70 \mathrm{mg}$ compared with once-weekly risedronate $35 \mathrm{mg}$ in women with postmenopausalosteoporosis: a randomized double-blind study. J Bone Miner Res, 20:141-51.

Saag KG, Emkey R, Schnitzer TJ, et al. 1998. Alendronate for the prevention and treatment of glucocorticoid-induced osteoporosis. $N$ Engl J Med, 339:292-9.

Sambrook PN, Geusens P, Ribot C, et al. 2004. Alendronate produces greater effects than raloxifene on bone density and bone turnover in postmenopausal women with low bone density:results of the EFFECT (Efficacy of FOSAMAX versus EVISTA Comparison Trial) international. J Int Med, 255:503-11.

Schnitzer T, Bone HG, Crepaldi G, et al. 2000. Therapeutic equivalence of alendronate $70 \mathrm{mg}$ once-weekly and alendronate $10 \mathrm{mg}$ daily in the treatment of osteoporosis. Aging Clin Exp Res, 12:1-12.

Tonino RP, Meunier PJ, Emkey R, et al. 2000. Skeletal benefits of alendronate; 7-year treatment of postmenopausal osteoporotic women. J Clin Endocrinol Metab, 85:3109-15.

Tonino RP, Santora AC, Ross PD. 2001. Safety of long term alendronate. J Clin Endocrinol Metab, 86:1835-6.

Van Staa TP, Leufkens HGM, Abenhaim L, et al. 2000. Use of oral corticosteroids and risk of fractures. J Bone Miner Res, 15:9931000.

Van Staa TP, Leufkens HGM, Cooper C. 2002. The epidemiology of corticosteroid-induced osteoporosis. Osteoporos Int, 13:777-87.

Walsh LJ, Wong CA, Pringle M, et al. 1996. Use of oral corticosteroids in the community and the prevention of secondary osteoporosis: A cross sectional study. BMJ, 313:344-6.

Wasnich RD, Miller PD. 2000. Antifracture efficacy of antiresorptive agents are related to changes in bone density. J Clin Endocrinol Metab, 85:231-6.

Wasnich RD, Bagger YZ, Hosking DJ, et al. 2004. Changes in bone density and turnover after alendronate or estrogen withdrawal. Menopause, 11:622-30.

Weinstein RS. 2001. Glucocorticoid-induced osteoporosis. Rev Endocr Metab Disord, 2:65-73. 
